\title{
A New Characterization Method for Delay and Power Dissipation of Standard Library Cells
}

\author{
JOS B. SULISTYO* and DONG S. HA ${ }^{\dagger}$ \\ Department of Electrical and Computer Engineering, Virginia Tech, Blacksburg, VA 24061, USA
}

(Received 15 March 2001; Revised 30 January 2002)

\begin{abstract}
A simplified method for characterization of standard library cells based on the linear delay model is presented in this paper. The linear model is chosen as it allows rapid characterization with a modest number of simulations, while achieving acceptable accuracy. All the parameters of cell delays are defined as 50\%-to-50\% delays, as distinguished from 50\%-to-threshold or threshold-to-50\% often used in commercial tools. We found that the 50\%-to-50\% definition of delays is more consistent and leads to closed-form formula. A subset of library cells in a $0.25 \mu \mathrm{m}$ technology was characterized using the proposed technique. A test circuit was subsequently generated and simulated to determine the accuracy of the proposed characterization method. SPICE simulations on the test circuit show that the timing estimations obtained through the proposed method is accurate to within $5.6 \%$, and the power estimation was accurate to $4.2 \%$, ignoring parasitics on interconnections.
\end{abstract}

Keywords: Standard cell; Characterization; Timing models; Linear model; Power estimation

\section{INTRODUCTION}

Automatic synthesis tools employ standard cell library based design. Facilitating logic optimization as well as speed and power estimation for a synthesis tool necessitates that cell libraries contain accurate information on the timing and power dissipation parameters. At the same time, the modeling of standard cells should be simple to avoid long processing time. In this paper, we present a method to characterize library cells in terms of delay and power dissipation.

Patel proposed a method to characterize cell delay and capacitance parameters based on a nonlinear lookup table method, and described a system implementing the method [7]. Patel's technique measures the delay as the switchingto-switching voltage. While the proposed definition may result in more accurate delay estimation for one kind of cells, it may lead to an inconsistent definition if a cell drives another cell of a different type (and hence, different switching voltage). Cirit proposed a slightly different method, one which slightly differs in that it assumes the cell being characterized as a black box (i.e. making no assumption about its internal structure) [3]. The black-box approach is largely adopted in our method due to its simplicity.
A nonlinear delay model based on a lookup table is generally more accurate, but its complexity slows down the logic simulation. Moreover, it needs intensive simulation of cells to obtain lookup table values. By contrast, the linear delay model, which is simple at the cost of lower accuracy, alleviates the problem and is often used for analysis of the synthesized circuit at the logic level. Since the focus of our work is to devise a simple, yet effective, delay and power characterization methods, we employed the linear timing model due to its simplicity and reasonable accuracy. Our linear delay model is largely based on Penfield-Rubenstein-slope model described in Eshraghian and Weste's book [4]. Readers may refer to Refs. [4,8] for the basics for cell delay and power dissipation models employed in our method. Recently, Auvergne proposed a method to improve the accuracy of the linear modeling at the cost of higher complexity [1].

All existing power dissipation models including our model are based on lookup tables. Cells were viewed mostly as black box entities for our method, except in the cases of simple combinational cells for which some input transitions are known to dissipate little internal energy. This means that, except for such simple cells, the characterization process will be more or less exhaustive. This approach is adopted because the

*E-mail: E-mail: jsulisty@ee.vt.edu

†Corresponding author E-mail: ha@vt.edu 
characterization task may not be performed by the designer of the cells. Additionally, the use of exhaustive input patterns should improve accuracy. However, knowledge about the internal structure of a cell allows the elimination of unnecessary test patterns. For more complicated cells and macrocells, some techniques to simplify characterization tables, such as those proposed by Jou et al. may be necessary [5,6]. Their techniques are particularly useful for cases where the internal structures of the cells are known, which is typically the case with most arithmetic macrocells.

The paper is organized as follows. The second section presents basics of the linear delay model and power estimation. Third and fourth sections describe proposed characterization techniques for timing and power dissipation, respectively. Fifth section shows experimental results on the proposed techniques and describes observations made from the experiments. Sixth section concludes the paper.

\section{PRELIMINARIES}

As previously mentioned, our work focuses on a threeterm linear model, which provides a reasonable tradeoff between simplicity and accuracy. In a linear model, the delay of a cell is modeled as a one- to three-term linear function of the load capacitance and the input ramp time. The simplest one-term linear model is

$$
\text { Delay }=k_{\mathrm{c}} C_{\mathrm{L}}
$$

where $C_{\mathrm{L}}$ is the load driven by the cell. This model is satisfactory for cells under heavy loading (i.e. the load is much greater than the input and output capacitance of the cell). When the cell is not heavily loaded, formula (1) is inaccurate. Note that when a cell is not driving any loads, it still exhibits a nonzero delay. Therefore, a two-term linear model is often used to improve the accuracy of the delay estimate.

$$
\text { Delay }=d_{0}+k_{\mathrm{c}} C_{\mathrm{L}}
$$

where $d_{0}$ is the delay of the cell under the load-free condition. The $k_{\mathrm{c}} C_{\mathrm{L}}$ term is called the transition delay [11] of the cell. Both of the one- and two-term linear models, however, ignore the effect of the nonzero input ramp times. Hence, a three-term linear model is expressed as:

$$
\text { Delay }=d_{0}+k_{\mathrm{c}} C_{\mathrm{L}}+k_{\mathrm{t}} t_{\mathrm{r}}
$$

where $t_{\mathrm{r}}$ is the ramp time of the input. The $k_{\mathrm{r}} t_{\mathrm{r}}$ term is called the slope delay [10].

The $k_{\mathrm{c}} C_{\mathrm{L}}$ term could be viewed as a RC delay exhibited by the cell. However, in two- and three-term models, the RC-delay corresponds to output ramp times rather than a $50 \%$-to- $50 \%$ delay of the cell. The $d_{0}$ term corresponds to the delay from input reaching $50 \%$ to output beginning to switch. It is proposed in this work that the terms are redefined to allow all the three delay components be measured as $50 \%$-to-50\% delays.

We equate the $50 \%$ point as the cell's switching point in our work, although this is not strictly true. Even ignoring process variations, the switching point depends on the cell type, and a closed-form formula to calculate switching points is not always possible. Hence, we employ $50 \%$-to$50 \%$ delays, whose reference points are $50 \%$ of the full swing voltage. While the physical importance of the $50 \%$ value is questionable, this leads to a more consistent definition of delays. Hereafter, we use "logical delay" and "50\%-to-50\% delay" interchangeably. The three important different delays are defined as follows. We refer to Fig. 1 in the description of the delays, and the four cells, Cell1 through Cell4, are identical in the figure.

Intrinsic delay $\left(d_{\mathrm{i}}\right)$ : The intrinsic delay is defined as a logical delay exhibited by a cell. The cell under consideration does not drive any load; instead, it is driven by other cells which themselves do not drive any load. Since the statement is contradictory, it is hypothetical. It is defined only to guarantee that its value is constant. Figure 1 shows the intrinsic delay of Cell2, which does not drive any load, but is driven by an identical load-free Cell1 indirectly through the voltage source.

Transition delay $\left(d_{\mathrm{t}}\right)$ : The transition delay is defined as an additional $50 \%$-to-50\% delay occurring when the cell under consideration drives a capacitive load. Cell3 in Fig. 1 exhibits the transition delay. For the linear model, the sensitivity of the delay of a cell to any capacitive load is characterized by its output resistance, as given in Eq. (4).

$$
\begin{aligned}
\text { Transition delay }= & \text { Output resistance } \\
& \times \text { Load capacitance }
\end{aligned}
$$

Slope delay $\left(d_{\mathrm{s}}\right)$ : When the less steep output voltage $V_{\mathrm{o} 3}$ in Fig. 1 drives Cell4, it incurs additional delay in addition to the intrinsic delay and the transition delay; this delay is defined as the slope delay.

The energy dissipated by a cell is the sum of the static energy and the dynamic energy. Static energy is dissipated by a cell when there is no logical transition on any input port. It is attributed to the leakage current for CMOS circuits. Dynamic energy, on the other hand, is dissipated by a cell that is undergoing one or more input transitions. The dynamic energy could be further divided into switching energy and internal energy. The switching energy is attributed directly with the rail-to-rail switching of the capacitive load at the output of the cell, which is obtained as $(1 / 2) C_{\mathrm{L}} V^{2}$ for each low-to-high or high-tolow transition. In the expression, $C_{\mathrm{L}}$ is the load capacitance, and $V$ is the supply voltage. The internal energy is the one dissipated internally, which is the difference between the dynamic energy and the switching energy. Note that the output resistance of a cell or the routing resistance is not considered explicitly in the 

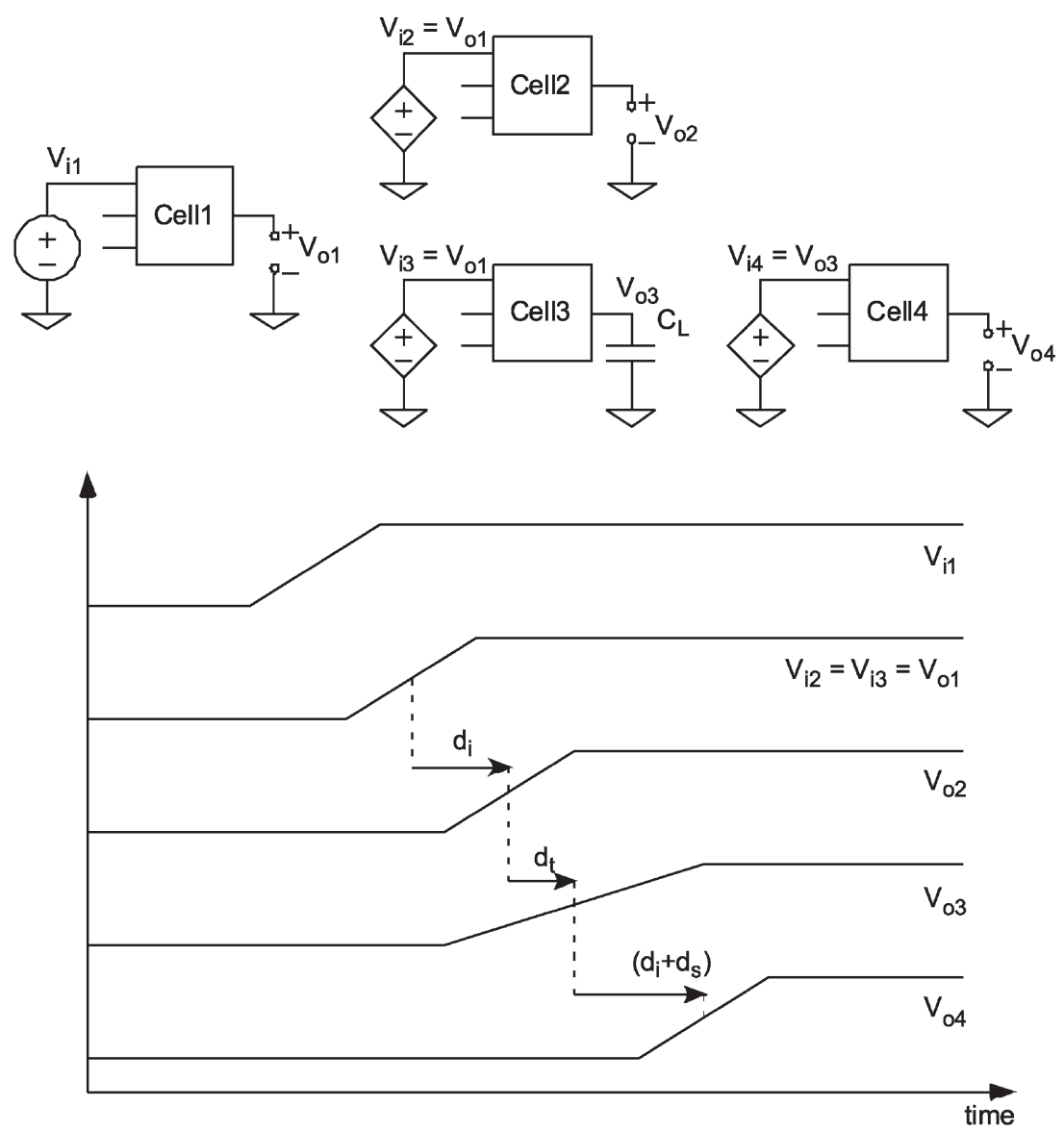

FIGURE 1 Intrinsic, transition, and slope delay.

energy dissipation of a cell. Therefore,

$$
\begin{aligned}
\text { Total energy }= & \text { Static energy }+ \text { Switching energy } \\
& + \text { Internal energy }
\end{aligned}
$$

Since the internal energy dissipation depends on both the input slope (represented by the input signal transition delay) and the load capacitance [12], the energy estimation process should be performed for various values of the input transition delay and the load capacitance. The results may be stored in a two-dimensional lookup table. However, for simplicity, the internal energy is often lumped and associated at input pins called input energy and/or at the output pin called the output energy. In such a case, only one-dimensional lookup table is required for each pin. Some CAD tools model output energy as a function of the load capacitance, and we adopt the approach in our model as well.

Power estimation typically calculates the energy first, and then divides the energy by the simulation time to obtain the average power. Hence, the energy dissipation of a cell is characterized for three parameters: static energy, switching energy, and internal energy. The static energy dissipation is different for high and low logic values at the output, while the internal energy dissipation is also dependent on rising and falling output transitions.

\section{PROPOSED TIMING CHARACTERIZATION TECHNIQUE}

In this section, we describe the estimation of resistance and capacitance of pins necessary to model transition and slope delays. We also present characterization of setup time and of tristate buffers. Finally, we discuss limitations of the proposed techniques.

\section{Resistance Estimation}

The transition delay $d_{\mathrm{t}}$ of a cell is modeled as follows in the linear model.

$$
d_{\mathrm{t}}=\text { Output resistance } \times \text { Load capacitance }
$$

The linear model gives one single value of the transition delay. However, the transition delay may be different for rising and falling transitions. Even for the same rising or falling transitions, it may be different depending on input pins. To address the problem, proper resistance values must be associated with each transition delay. There needs to be one resistance value for each pair of an output transition (rising or falling) and an input pin. 


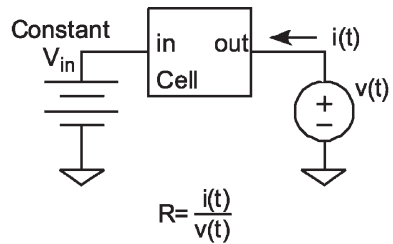

(a) physical resistance

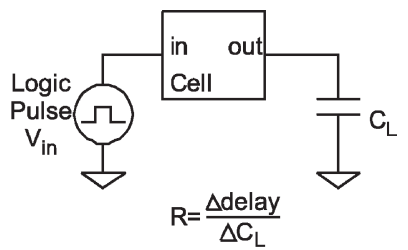

(b) proposed resistance
FIGURE 2 Estimation of output resistance.

The physical definition of output resistance, which is unique and constant for the output pin, is obtained as

$$
R_{\text {out }}=d\left(I_{\text {test }}\right) /\left.d\left(V_{\text {test }}\right)\right|_{V_{\text {in }}=\text { constant }}
$$

In contrast, for our model, the resistance is estimated as

$$
R_{\text {out }}=\Delta(\text { Delay }) / \Delta(\text { Capacitance })
$$

The setup to estimate output resistance is shown in Fig. 2. It should be noted that several commercial tools employ separate pairs of rising and falling resistance values for each combination of input and output pins.

\section{Slope Sensitivity Estimation}

A cell exhibits the slope delay $d_{\mathrm{s}}$ when it is driven by another cell with a transition delay. Once a transition delay is estimated, the output slope delay and input transition delay are related as given in Eq. (8).

$$
d_{\mathrm{s}}=\text { Input signal transition delay } \times \text { Slope sensitivity }
$$

The slope sensitivity is distinct for each input-output pair delay, and is obtained from a test input transition and the resultant output slope. It should be noted that a cell exhibits both transition and slope delays. The separation is made only for the mathematical convenience of the estimation during simulation.

For sequential cells and complex combinational cells, the requirements that the driving cell must be of the same type may not hold well. For instance, a flip-flop may be driven by an inverter or a clock driver.

\section{Capacitance Estimation}

Other parameters that need to be characterized are dynamic input capacitance for all cells and output capacitance for only tristate cells. The output capacitance of tristate cells need to be characterized, since tristate buffers are usually tied together, with several tristate cells driving the same output node, but with only one, or none at all, being enabled at any given time. In that case, the output capacitance of the disabled tristate cells poses a capacitive load to the enabled tristate cell and hence increases the transition delay of the enabled cell. Even if nontristate cells are tied together (such as parallel buffers driving heavy loads), they are designed to have the same logic state. The effect of their output capacitance on delays is absorbed into the intrinsic delay term in Eq. (4).
The capacitance of a port is expressed as:

$$
C=Q / V=\frac{1}{V} \int_{0}^{t} i(t) \mathrm{d} t
$$

where $V$ is the rail-to-rail voltage and $i(t)$ is the current flowing into the node. It is necessary to set reasonable rise/fall time of the applied voltage $V$. It is set to $0.4 \mathrm{~ns}$ for the $0.5 \mu \mathrm{m}$ technology and $0.2 \mathrm{~ns}$ for $0.25 \mu \mathrm{m}$ technology for our method for both rise and fall times. Most SPICElike simulators have measurement statements that perform an integral operation. Such a measurement statement can be used to perform the necessary integral operation of current $i(t)$ in Eq. (9).

Since the value of the dynamic capacitance may vary depending on the particular transition, we propose the measurement be performed for all input transitions causing output transitions for combinational cells. This may result in a reasonably pessimistic estimate, i.e. a larger capacitance value. When an input change causes the output change, the effective input capacitance increases slightly due to the Miller effect. For sequential cells, however, this requirement does not apply, since some inputs may not affect the outputs immediately (e.g. the change in the D input of a D flip-flop does not cause the output change). Further, the dynamic capacitance not only depends on an input transition, but also the state of the clock. Therefore, it is prudent that all cases be tested and the results be averaged.

For instance, we explain the capacitance estimation of a rising-edge triggered D flip-flop. Four different cases as given in Table I should be considered for the capacitance estimation of $\mathrm{D}$ input.

The results of Case 1 and Case 2 are averaged to estimate the input capacitance of the $\mathrm{D}$ input under clock $=0$, while the results of Case 3 and Case 4 are averaged to obtain the input capacitance of the D input for clock $=1$. The final estimate is the larger of those two cases. Similarly, the capacitance of all the inputs other than the clock can be estimated. The capacitance for the clock input is measured for rising clocks for four different cases of the $Q$ value as described below, and the average value is taken as the estimate.

i) $Q$ changes from 0 to 1

ii) $Q$ remains high.

iii) $Q$ changes from 1 to 0 .

iv) $Q$ remains low.

TABLE I Capacitance estimation for D input

\begin{tabular}{lcc}
\hline Case & D input & Clock \\
\hline 1 & Rising & 0 \\
2 & Falling & 0 \\
3 & Rising & 1 \\
4 & Falling & 1 \\
\hline
\end{tabular}




\section{Setup and Hold Time Estimation}

A typical definition of the setup time, i.e. the time required to guarantee the correct operation of a flip-flop, is too loose to be used for characterization. Instead, we use a stricter definition of the setup time based on earlier works [9,11]. The setup time of a flip-flop is the time clearance between the D input and the clock edge such that any later arrival of the input signal would incur at least $30 \%$ of additional delay to the reference clock-to-output delay. The reference clock-to-output delay is the one in which the input signal is applied sufficiently earlier than the clock edge. It is possible that the setup time can be a negative value. Since various synthesis or high-level timing estimation tools cannot properly deal with negative setup times, negative setup times are replaced with zero.

The hold time is defined in a similar manner as setup time, i.e. the clearance after the clock edge for the input such that if the input comes any earlier, the clock-tooutput delay increases by $30 \%$ or higher above the reference case. In general, hold time needs to be characterized only if it is at least as long as the clock-tooutput delay. For master-slave structures including most of the popular static CMOS implementations, the case seldom occurs. Hence, the hold time was not characterized in our work.

The procedure to measure the setup time is as follows. First, apply the input well ahead of the clock edge and measure the reference clock-to-output delay. Next, vary the transaction time of the input until the clock-to-output delay is 30\% longer than the reference delay. The optimization feature of HSPICE, for instance, could be used for this purpose. The clock rise/fall time should be made reasonably slow to guarantee a reasonably pessimistic estimate. We used rise/fall times of $0.5 \mathrm{~ns}$ for $0.5 \mu \mathrm{m}$ technology and $0.4 \mathrm{~ns}$ for $0.25 \mu \mathrm{m}$ technology, respectively, both for the clock and for the $\mathrm{D}$ input.

\section{Tristate Buffers and Inverters}

Tristate cells are basically characterized in the same way as other combinational cells except for three considerations, namely enable/disable delays, charge injection, and output capacitance. The three considerations are described in the following three sections.

\section{Enable and Disable Delays}

Enable and disable delays between the enable signal and the output are defined as follows:

- Enable-rise delay: Enable signal to the Z-to-0 transition at the output.

- Enable-fall delay: Enable signal to the Z-to-1 transition at the output.

- Disable-rise delay: Enable signal to the 0-to-Z transition at the output.

- Disable-fall delay: Enable signal to the 1-to-Z transition at the output.
It should be noted that several synthesis tools may not define disable delays under the assumption that disable delays are always shorter than enable times. Hence, they are not observed nor have any effects during the normal circuit operation.

\section{Charge Injection}

The charge injection phenomenon makes a tristate buffer/inverter difficult to characterize in isolation; rather, two or more cells need to be characterized while driving the same node. Consider the tristate buffer and waveforms shown in Fig. 3. The circuit enclosed by the dashed line in Fig. 3(b) denotes the tristate output stage. When $V_{\text {en }}$ is deasserted at $2.5 \mathrm{~ns}, V_{\text {out }}$ is left floating, and the charge accumulated at the channels of $Q_{\mathrm{P} 2}$ and $Q_{\mathrm{N} 1}$ are dumped into the node $V_{\text {out }}$. Therefore, the voltage $V_{\text {out }}$ exceeds the rail voltage at $3 \mathrm{~ns}$ and cannot be used to characterize a propagation delay of logic values. Note that voltage levels of signals should stay within the rails to be used for a logic delay characterization.

In order to address the problem, we propose a parallel connection of two buffers which are driven by opposite logic values as shown in Fig. 4(a). All the five buffers are identical. One buffer in the circuit is always active, so that the node $V_{\text {op }}$ is limited to the rail voltage. The remaining problem is how to obtain the intrinsic delay $d_{\mathrm{i}}$ of the buffers. Since a buffer is a load of the other buffer for the circuit in the figure, the propagation delay $\Delta_{1}$ measured from the circuit is the case with one buffer. Consider the

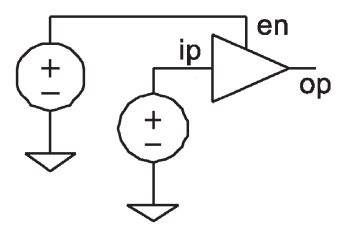

(a) external circuit connection

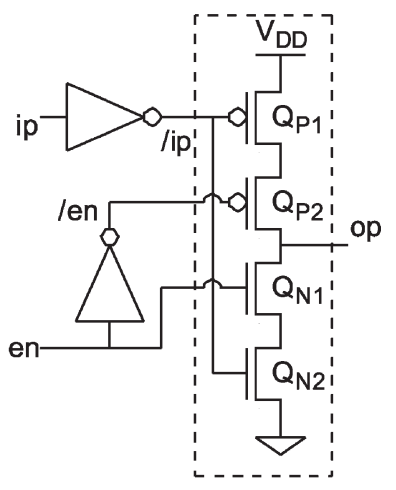

(b) internal circuit

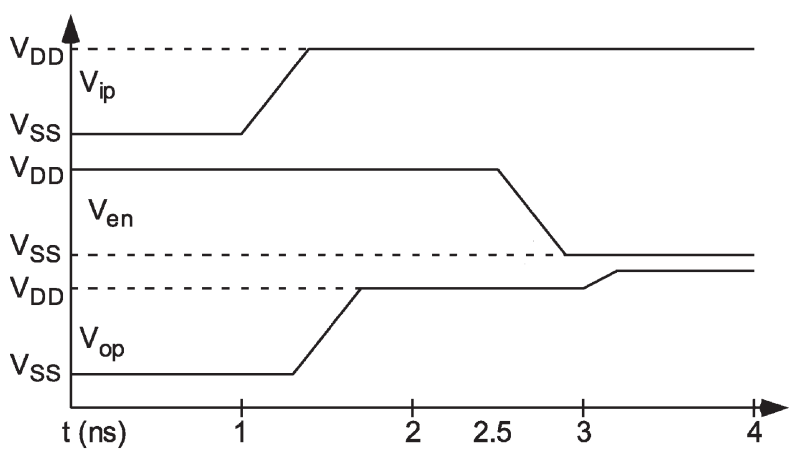

(c) wave form showing effects of charge injection

FIGURE 3 Tristate cell exhibiting charge injection condition. 


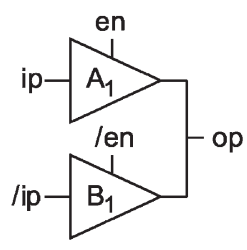

(a) $A_{1}$ drives an effective load of $B_{1}$ output capacitance

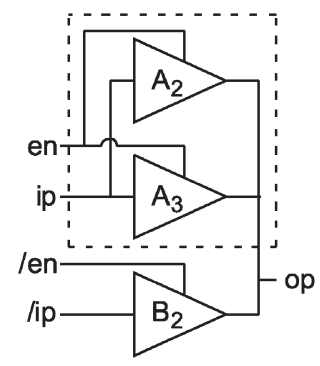

(b) $A_{2}$ and $A_{3}$ each drives a load of $\frac{1}{2} \times$ the output capacitance of $B_{2}$

FIGURE 4 Delay determination of tristate circuits.

circuit shown in Fig. 4(b), in which the circuit inside the dashed line is a true input block. When $A_{2}$ and $A_{3}$ buffers switch simultaneously, the effective load is a half of $B_{2}$ buffer. Let us denote $\Delta_{2}$ as the propagation delay of the circuit. The difference $\Delta_{1}-\Delta_{2}$ is the transition delay due to a half load of $B_{2}$ buffer assuming the transition delay is proportional to the load. Under the assumption, the intrinsic delay (which is the delay without any load) is obtained as $\Delta_{2}-\left(\Delta_{1}-\Delta_{2}\right)$ or $2 \Delta_{2}-\Delta_{1}$.

The above parallel circuits in Fig. 4 can also be used for resistance estimation. Consider the estimation of the output resistance $R_{\text {out, normal }}$ on the normal input (i.e. $V_{\text {ip }}$ node in Fig. 4) of an enabled buffer. Suppose that a capacitive load $C_{\mathrm{L}}$ is attached at the output of the circuit in Fig. 4. Let us denote $D_{\mathrm{L}}$ as the propagation delay with the capacitive load and $\Delta_{1}$ the propagation delay without the capacitive load (i.e. the original circuit). Then, $R_{\text {out }}$ on the normal input is obtained as $\left(D_{\mathrm{L}}-\Delta_{1}\right) / C_{\mathrm{L}}$. We defer the estimation of output resistance on the enable input to the next section.

The estimation of input capacitances of tristate buffers also uses the parallel circuit in Fig. 4. It is important to note that the input capacitance is estimated only for the enabled buffer of the parallel circuit, since the disabled one does not make the output change. The input capacitance estimation for the normal input $V_{\mathrm{i}}$ of the enabled buffer is the same as other cells described in the section on "Capacitance Estimation". There are four possible cases for the enable input, namely, enable-rise, enable-fall, disable-rise, and disable-fall (refer to "Enable and Disable Delays" Section). The estimated value is the average of the four capacitances obtained from the four cases.

\section{Output Capacitance}

The outputs of tristate buffers/inputs may be tied together, and inactive ones exhibit output capacitances to the active one. Therefore, it is necessary to consider the output capacitance of an inactive buffer/inverter, which is a unique feature of tristate cells. The estimation procedure is the same except the voltage source is applied to the output of an inactive cell under the normal input $V_{\mathrm{i}}=0$ and 1 .

The output resistances $R_{\text {out, enable }}$ on the enable input can use the output capacitance obtained above. The propagation delay for the parallel circuit in Fig. 4(a) is estimated by applying an enabling input under the normal input $V_{\text {ip }}=0$ and $V_{\text {ip }}=1$. By subtracting intrinsic delay $d_{\mathrm{i}}$ (obtained earlier) from the propagation delay, the transition delay $d_{\mathrm{t}}$ is obtained. Further, the output resistance is computed as $R_{\text {out, enable }}=d_{\mathrm{i}} / C_{\text {out }}$, where $C_{\text {out }}$ is the output capacitance. The disable delay was not measured in our work, as the disabling process is not directly observable in terms of logic values. We assume that the disabling process is sufficiently fast to avoid conflicts on logic values.

\section{Limitations and Problems}

The main problem of the proposed method lies in the slope sensitivity. We assumed that the slope delay is linear to an input slope. However, the assumption fails for extreme cases. For example, if the input waveform is very slow, the total delay-including slope delay-is negative [8]. In fact, some cells exhibit negative slope dependency for the input range in our works.

Another problem arises if the cells being characterized display a considerable variation in output slope even under the loadless condition. In such a case, the driving cell should be ideally an "average" cell instead of the identical cell. Although it is surmised that the error resulting from our approach should average out to zero for a large cell library, it may pose some problem for significantly different types of cells (such as an inverter drives a sixinput NOR gate).

Finally, the proposed approach becomes complex for functionally complicated cells with large numbers of input-to-output paths. In such a case, one may select some typical transitions. Finding what would constitute typical transitions, however, is beyond the scope of our work.

\section{PROPOSED POWER CHARACTERIZATION TECHNIQUE}

There are three parameters relevant to power estimation, namely input/output capacitance, internal energy, and static power. Since determination of capacitances is already performed during timing characterization, we discuss characterization techniques for the latter two parameters in this section.

\section{Static Power Estimation}

We adopted the following static power estimation method. The measurement of static power should be performed long after all transients have settled down. For most cells, a wait time of $20 \mathrm{~ns}$ (after the last input transition) should be adequate. 
- For a relatively simple combinational cell, the power is estimated for all possible input combinations, and averages are calculated for both output high and low. It is assumed that all inputs are equally likely in this approach

- For sequential cells, the estimation is made for both output high and low, using only "normal mode" operations-for instance, only the D input and the clock input for a D flip-flop. The set/reset inputs and the scan operation should not be used, as they are inactive during the normal operation.

It should be noted that the static power dissipation is insignificant unless the circuit is likely to stay dormant for a long time. Hence a characterization may not be needed at all for most cells and operating conditions.

\section{Internal Energy Measurement}

The internal energy is measured by effecting a logic transition on a cell's input and by measuring the resulting energy dissipation by the cell. Only one input should change at any given time. For modeling purposes, the internal energy is lumped into each of the cell's input and output pins, instead of the entire cell. For ease of mathematical analysis, internal energy is divided into two kinds:

i) input energy, representing the internal energy dissipated by a cell due to input transitions which do not cause output changes, and;

ii) output energy, representing the additional internal energy caused by output transitions.

The input energy is lumped into the input pins, while the output energy is lumped into the output pins. Hence, if an input change causes an output change, it will dissipate not only the input energy (due to the input change), but also the output energy (because of the output change); and, if a load capacitance is present, also the switching energy, (because the output change also causes the charging or discharging of the load capacitance). However, if the input change causes no output change, only the input energy is dissipated.

\section{Internal Energy Characterization for Simple Combinational Cells}

Typically, input energy is measured first, and then the output energy is obtained by subtracting the appropriate input energy from the total internal energy. Note that the total internal energy is obtained from subtracting the switching energy from total energy dissipation over a certain period. Also, it may be sufficient to consider only one input change at any given moment; it may be unnecessary to consider simultaneous input changes, as the increase in accuracy is limited. It also complicates the mathematical model for parameter extraction.

\section{Internal Energy Characterization for Combinational and Tristate Cells}

For simple combinational cells, input energy is negligible; the cells dissipate substantial dynamic energy only if their outputs actually change. The internal energy of a cell is a function of the input signal transition delay and of the load capacitance. Generally, the dependence is stronger towards the input slope; however, with some certain tools, it is more natural or even required to associate the output energy with the load capacitance. For such cases, one needs to choose a typical input slope, and then vary the load capacitance.

For instance, the circuit and waveform in Fig. 5 was used in our characterization of a three-input NAND gate. The NAND gates inside the dashed lines degrade the input transition to represent a typical case, and the shaded NAND gate is another load of the circuit. We assumed that a typical load is two. The load capacitance of the cell being evaluated, meanwhile, was varied between 0 and 15 times the input capacitance. This is an adequately wide range.

For combinational cells with internal loads (such as a complementary CMOS multiplexer or an AND-ORINVERT gate) or sequential cells, the internal energy may be dissipated even if no output transition actually occurs. For such a cell, it is prudent to estimate the average energy for all possibilities. Therefore, to compute the internal input energy, we apply all possible input combinations, or a subset of them that does not change the output. We then integrate the power dissipation.

To compute the internal output energy, we apply input combinations which change the output and measure the energy dissipated until all transients have settled out, say $E$. The output energy is obtained by subtracting the input energy and the switching energy at the load $(1 / 2) C_{\mathrm{L}} V^{2}$ from $E$.

Tristate cells are characterized in the same way as complex combinational cells, except we should consider enable/disable energy and the tristate cells are tied together. As an example, the circuit and waveforms in Fig. 6 shows the characterization of noninverting tristate buffers. The same values of input transition delays and load capacitances as used in the D flip-flop characterization was used again here. The total capacitive loads of the buffers also include those buffers' own output capacitances.

\section{Internal Energy Characterization for Sequential Cells}

We describe the power estimation of sequential cells. We consider only one input change at any given time, and treat the clock as an input.

1. Estimate the clock energy, i.e. the internal energy associated with the clock change without any associated output change. The clock energy may vary for rising and falling transitions and also depends 

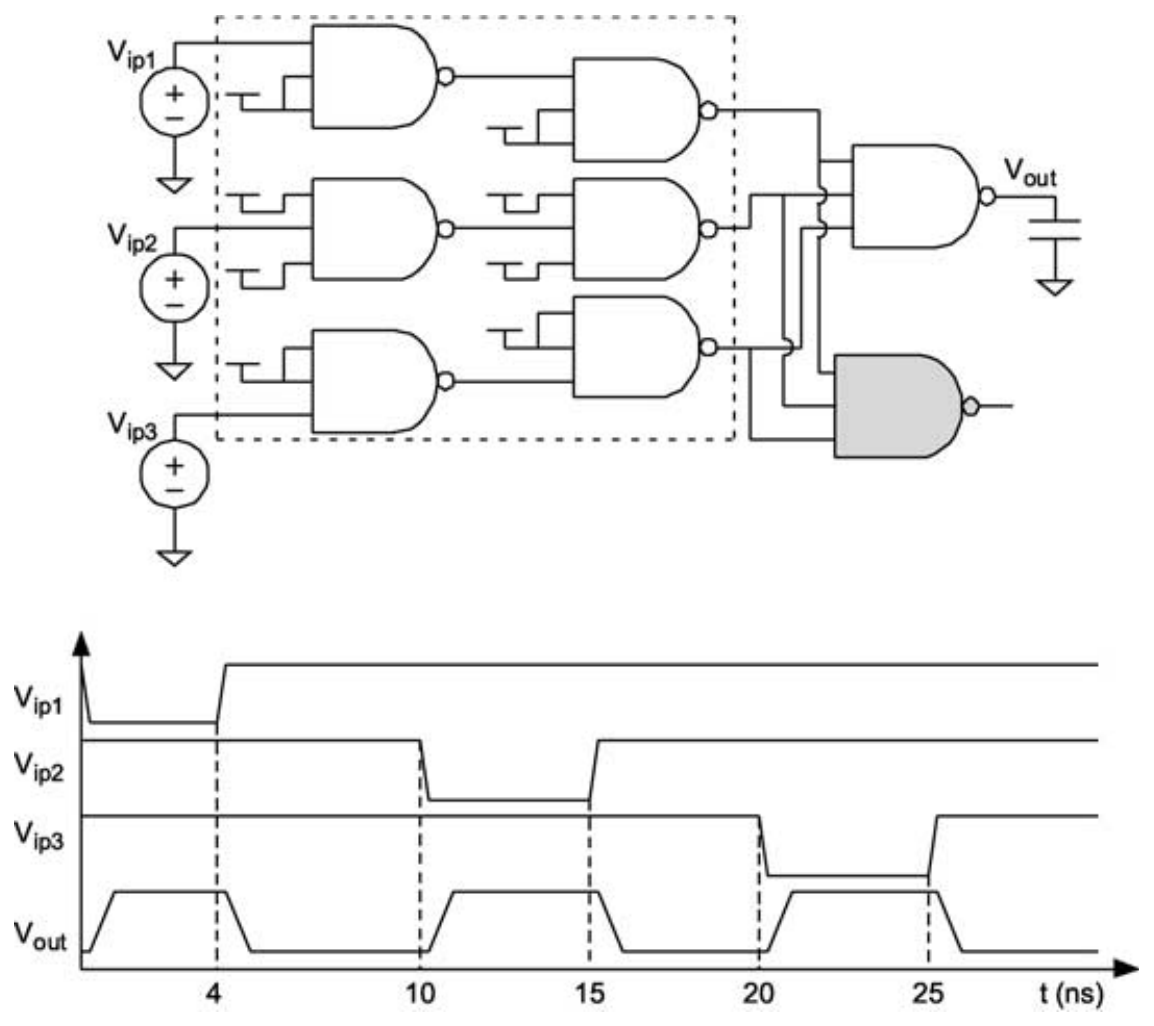

FIGURE 5 Power characterization of a 3-input NAND.

on the logic values at the outputs. One value of the clock energy is usually sufficient, using typical rise/fall-time and typical loads on the flip-flop's outputs.

2. Estimate the input energy of synchronous inputs, i.e. the internal energy caused by input transitions which do not cause any output change, such as D input for a D flip-flop. The input energy may have different values depending on whether the clock is high or low.

3. Estimate the output energy for cases where the output transition is caused by a clock transition.

4. Estimate the output energy due to asynchronous inputs such as asynchronous reset or set inputs.

The above sequence was chosen deliberately to avoid any important energy, such as the clock and the synchronous input/output, from having negative values. As the possible orders in the sequence are not unique, the energy values are also not unique either.

The power characterization of a rising-edge triggered $\mathrm{D}$ flip-flop with asynchronous reset is shown in Fig. 7. The clock and input slope variations were generated by varying the load capacitance of inverters at $(0-20) \times$ typical pin capacitance range, while the load capacitance of the flipflop was varied in the $(0-10) \times$ typical pin capacitance range. The different ranges were chosen since it was surmised that the quality of the clock signal is likely to be rather poor in a cell-based design. It is assumed that the clock period is sufficiently long that the transients decay within $(1 / 4) \times$ clock period $T$ from the time that the stimuli are first effected. Therefore, the toggle energies are obtained by integrating the flip-flop's power dissipation within an integration period of (1/4)T. The sequence used for our method is as follows:

1. Measurement of the clock energy. The power dissipation is integrated between $n T$ and $(n+1 / 4) T$. Four cases of clock fall energy are considered at $n=1$, 2,3 , and 4 , for $\mathrm{DQ}=00,10,11$, and 01 , respectively. The clock fall energy is the average of the four cases. Two cases are considered for the clock rise energy, namely the $Q=0$ and $Q=1$ at $1.5 T$ and at $3.5 T$, and the average of the two cases is taken. If one chooses to simply use the average clock energy, the value used would be the average of the clock rise and the clock fall energy. The four time intervals for power measurements are shaded on the D input in the figure.

2. Measurement of the D energy. Four cases, rise/fall transition on $\mathrm{D}$, under the clock $=0$ or 1 , should be considered.

3. Measurement of the $\mathrm{Q}$ energy. Both $\mathrm{Q}$ rise and fall energy should be measured. $P\left(x_{\mathrm{ff}}\right)$ is integrated to obtain $E\left(x_{\mathrm{ff}}\right)$. For instance, the rise energy of $Q$ is $E\left(Q_{\text {rise }}\right)-(1 / 2) C_{\text {load }} V^{2}-$ (rising clock energy),

where $E\left(Q_{\text {rise }}\right)$ is the measured energy dissipation of the flip-flop.

4. Measurement of the set/reset energy. It should be measured separately for rising and falling cases. For the reset operation, it should measure four cases under $Q=0,1$ and clock $=0,1$. The four durations are gray in the waveform in Fig. 7. This is also true for the set operation. 

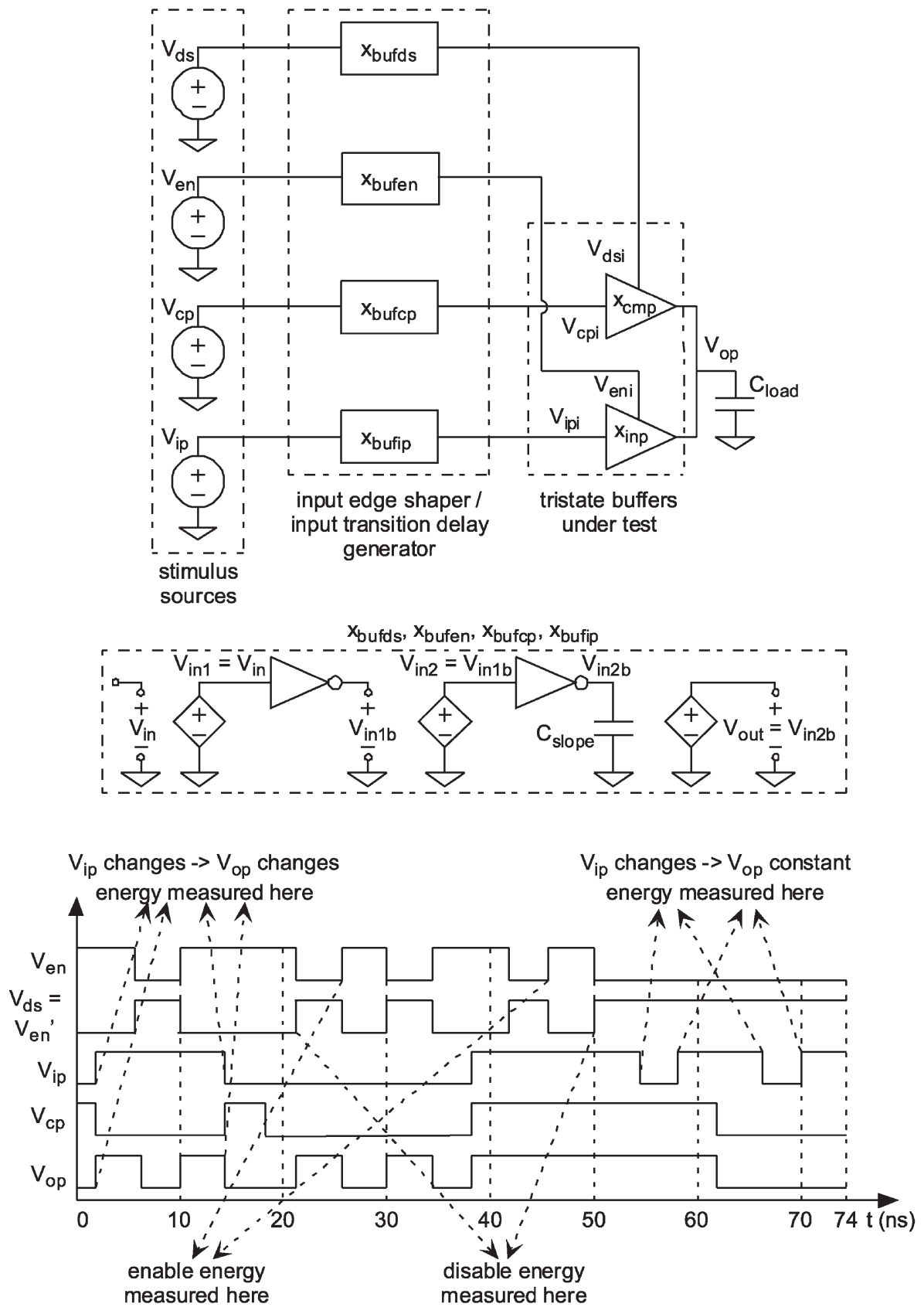

FIGURE 6 Circuit and waveforms for power characterization of tristate buffers.

Depending on the structure of the flip-flop, some of the above measurements may be unnecessary; for instance, for a rising-edge triggered master-slave D flip-flop, the master is inactive when the clock is high, and hence input (D) energy would be 0 when clock is high and need not be measured. Further, if a lower accuracy is deemed acceptable, some of those measurements, such as the reset energy, could be omitted as well.

\section{Limitations of the Power Measurement Technique}

The main problem for the proposed approach is the possibility that some of the energy will be found negative.
For example, the output energy of the flip-flop is calculated by subtracting the clock energy and load switching energy from the total energy dissipation of the flip-flop. While this is conceptually correct, it could result in negative output energy. Most simulation-based power estimation tools require that all energy be nonnegative. In that case, the energy should be replaced with zero, and this increases the inaccuracy. Another limitation of the proposed approach is that the accuracy may suffer for functionally complicated cells due to the lack of a systematic selection mechanism of test patterns. In that case, one may use the structure information of a cell to obtain a reduced set of characterization pattern $[2,5,6]$. 


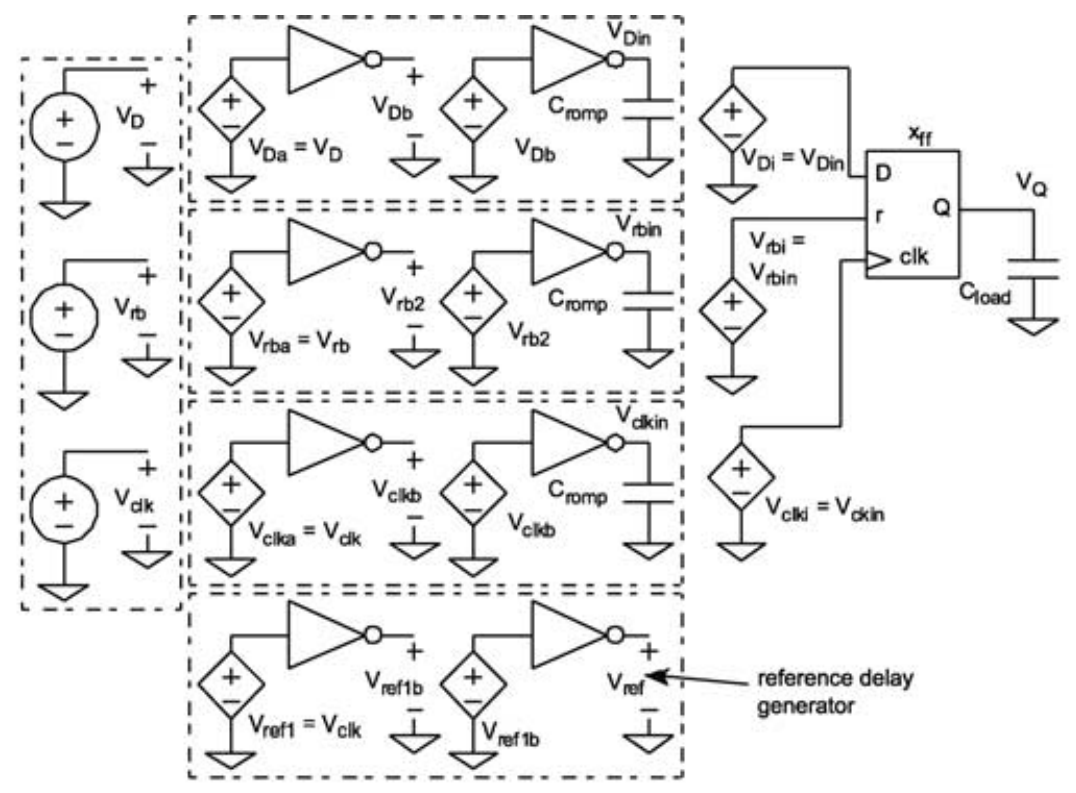

(a) circuit

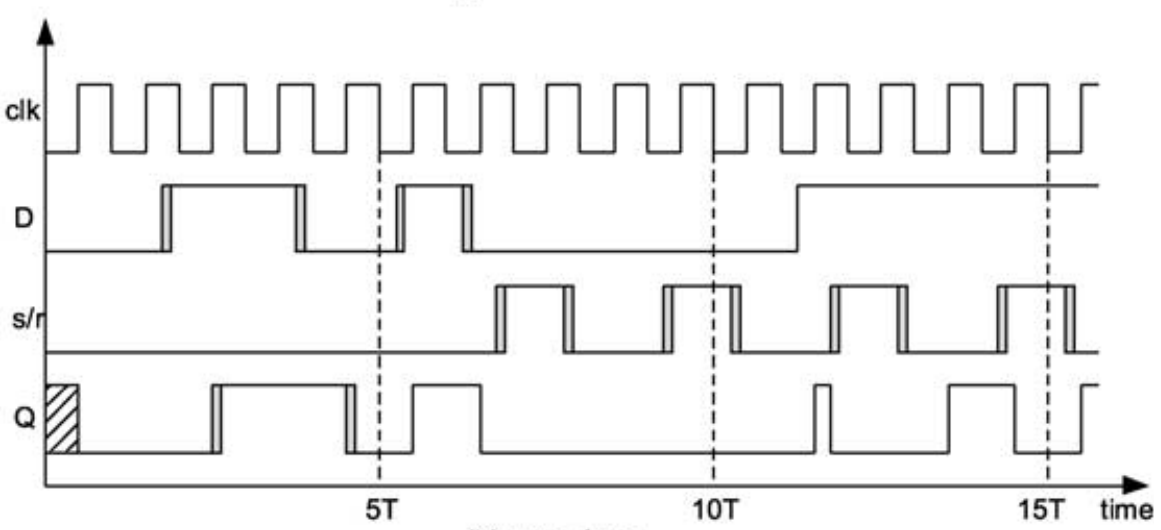

(b) wave form

FIGURE 7 Circuit and waveform for power measurement for a rising-edge triggered D flip-flop with high-active reset/set.

\section{EXPERIMENTAL RESULTS AND DISCUSSIONS}

As previously mentioned in "Limitations and Problems" Section, a problem with the proposed model is slope sensitivity. The dependence of the slope delay on the input transition delays is modeled as linear in our method, but it may not hold well for a wide range of input transition delay.

The circuit in Fig. 8(a) was used to experiment the accuracy of the proposed method for slope delays. The delay of the gray 2-input NAND gate was measured and compared with the prediction of our model. The input transition delay was varied by varying $C_{\mathrm{L} 2}$ in the $0-50 \mathrm{fF}$ range, where the typical load of an input pin is about $5 \mathrm{fF}$. Two different values of $C_{\mathrm{L} 3}, 5 \mathrm{fF}$ for a light load and $50 \mathrm{fF}$ ( $\approx$ fan-out of 10 ) for a very heavy load were used. The error is defined as:

Error $=[1-($ Predicted delay $/$ Measured delay $)]$
Figure 8(b) and (c) shows the experimental results, the worst inaccuracy attained for the rise delay is about $7.3 \%$ for the light-load and about $11.6 \%$ for the heavy-load. For fall delay, the accuracy is $6.8 \%$ for the light load and $8.9 \%$ for the heavy load. In both cases, the worst-case error was negative, signifying that the prediction of our model is somewhat optimistic. However, despite the simplified assumption of linearity, our method attains a reasonably good accuracy.

To evaluate the accuracy of our model on real circuits, we experimented with a circuit of two identical 4-bit counter in a $0.25 \mu \mathrm{m}$ technology. The circuit was described in VHDL, synthesized using a library characterized with our method and then laid out using a placement and routing tool. Although the two counters are identical in the VHDL description, the two counters are slightly different in delay and power dissipation due to differences in wire loads. Hence, the slower one dictates the critical path delay. We experimented with three different versions of the netlists of the circuit. The first one is the gate level netlist, in which the delay and power 


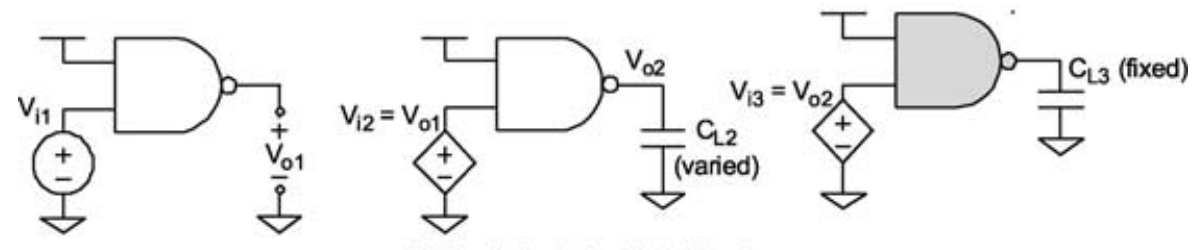

(a) Circuit for Delay Model Testing

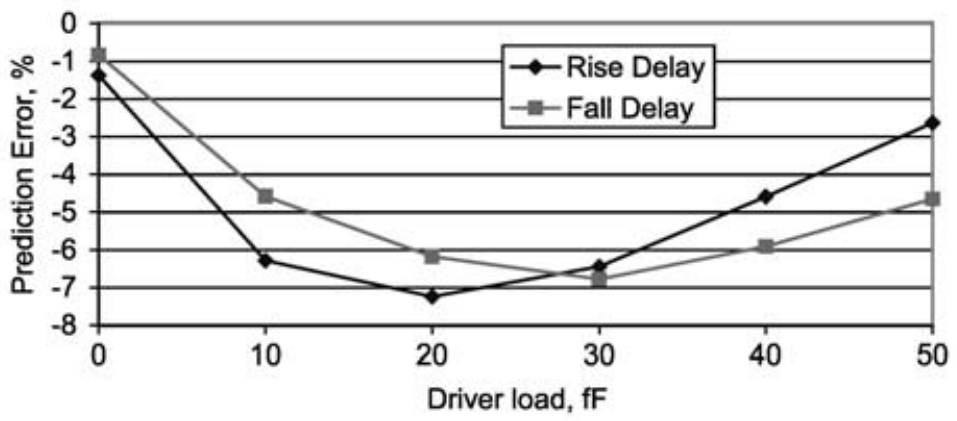

(b) Prediction error with $\mathrm{C}_{\mathrm{L} 3}=5 \mathrm{fF}$

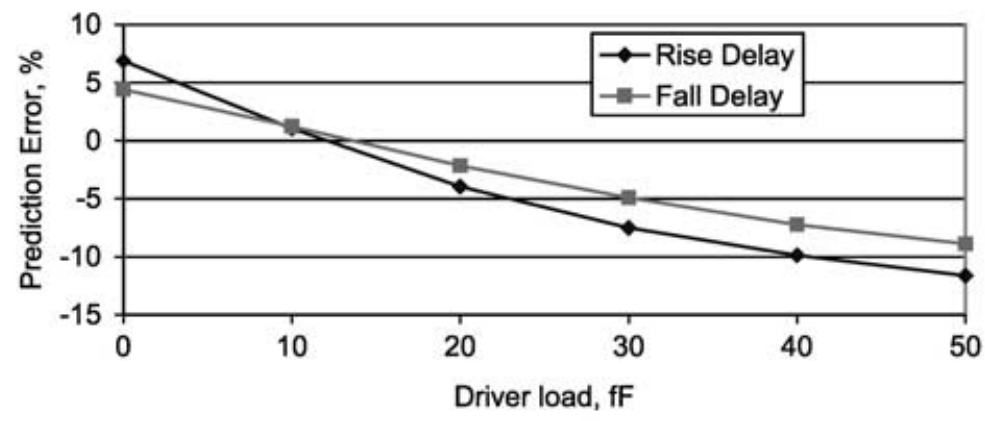

(c) Prediction Error With $\mathrm{C}_{\mathrm{L} 3}=50 \mathrm{fF}$

FIGURE 8 Prediction error for the proposed delay model.

dissipation parameters of gates were obtained using the proposed model. The gate level netlist does not contain parasitic wire delays. The second one is the transistor netlist extracted from the layout. The netlist contains parasitic capacitance on wires. It should be noted that our tool does not extract parasitic resistance. The third netlist is the same as the second one except the parasitic.

The critical path delay for the gate level circuit was given by a commercial synthesis tool. Further, HSPICE simulations were performed to obtain the critical delays and power estimation for the two transistor netlists. All simulations for power estimation of the circuits were performed for the clock running at $100 \mathrm{MHz}$. Rise and fall times of $0.4 \mathrm{~ns}$ were used for HSPICE simulation, while gate level simulations used zero-delay model, as it is the typical setting for large circuits.

The simulation results on critical path delays and power estimation is shown in Table II. The critical path delay based on our model is $1.52 \mathrm{~ns}$, while it is $1.74 \mathrm{~ns}$ for SPICE simulation with capacitive parasitics and $1.44 \mathrm{~ns}$ without capacitive parasitics. Since the gate level netlist based on our model does not consider the wire load, it may be fair to compare our model with the HSPICE simulation without the parasitic capacitances of the wire load. In such a case, the error of our model is $5.6 \%$, which is fairly accurate. The accuracy of the proposed model for power estimation is $17.9 \%$ compared with SPICE simulation with parasitics and is reduced to $4.2 \%$ without parasitics.

TABLE II Critical path delay and power estimates

\begin{tabular}{lccc}
\hline & Gate-level netlist & Transistor-level netlist with capacitive parasitics & Transistor-level netlist without capacitive parasitics \\
\hline Critical path delay & $1.52 \mathrm{~ns}$ & $1.74 \mathrm{~ns}(-12.6 \%)$ & $1.44(5.6 \%)$ \\
Power dissipation & $543 \mu \mathrm{W}$ & $661 \mu \mathrm{W}(-17.9 \%)$ & $567 \mu \mathrm{W}(-4.2 \%)$ \\
\hline
\end{tabular}


Again, our model for power estimation is fairly accurate compared with SPICE simulation without parasitics. Based on the two experiments, we conclude the following ones. (i) Our model is fairly accurate for both delay and power estimation. (ii) Characterization of the wire load through back annotation is essential to increase accuracy on both delay and power estimation.

\section{SUMMARY}

In this work, we proposed a method to characterize delay and power dissipation of cells based on the linear timing model. Our experimental results performed on 4-bit counters show that the error on the critical delay for the proposed method is $5.6 \%$ compared with SPICE simulation without considering parasitic capacitance on wires and the error on power estimation is $4.2 \%$. The accuracy of our model is sufficiently good to be practical. The experiment reveals that consideration of parasitic capacitances through back annotation is sensitive to delay and power estimation at the gate level. Further research is necessary in this area.

\section{Acknowledgements}

The authors would like to thank the current and former members of the Virginia Tech VLSI for Telecommunications (VTVT) Group at the Bradley Department of Electrical and Computer Engineering, Virginia Polytechnic Institute and State University, especially, Han Bin Kim, Suk Won Kim, Carrie Aust, Meenatchi Jagasivamani, Jia Fei, Nate August, Steve Richmond, and Andrew Gouldey, for all their help and suggestions along the course of this work.

\section{References}

[1] Auvergne, D., Daga, J.M. and Rezzoug, M. (2000) "Signal transition time effect on CMOS delay evaluation", IEEE Transactions on Circuits and Systems-I: Fundamental Theory and Applications 47(9), 1362-1369.

[2] Cheng, M., Irwin, M., Li, K. and Ye, W. (1999) "Power characterization of functional units", Conference Record of the Thirty-Third Asilomar Conference on Signals, Systems, and Computers, IEEE 1, 775-779.

[3] Cirit, M.A. (1991) "Characterizing a VLSI standard cell library", Proceedings of Custom Integrated Circuit Conference, IEEE, 25.7.1-25.7.4

[4] Eshraghian, K. and Weste, N.H.E. (1994) Principles of CMOS VLSI Design: A System Perspective (Addison-Wesley, Reading, MA).

[5] Jou, J.Y., Lin, J.Y. and Shen, W.Z. (1999) "A structure-oriented power modeling technique for macrocells", IEEE Transactions on VLSI 7(3), 380-391.

[6] Jou, J.Y., Lin, J.Y. and Shen, W.Z. (1990) "A power modeling and characterization method for the CMOS standard cell library",
Digest of Technical Papers, International Conference on Computer Aided Design, IEEE, 400-404.

[7] Patel, D. (1990) "CHARMS: characterization and modeling system for accurate delay prediction of ASIC designs", Proceedings of Custom Integrated Circuit Conference, IEEE, 9.5.1-9.5.6.

[8] Shoji, M. (1988) CMOS Digital Circuit Technology (Prentice-Hall, Englewood Cliffs, NJ).

[9] Stojanovic, V. and Oklobdzija, V.G. (1999) "Comparative analysis of master-slave latches and flip-flops for high-performance and lowpower systems", IEEE Journal of Solid-State Circuits 34(4), $536-548$.

[10] Synopsys Inc., Library Compiler User Guide, Vol. II, Chapters I-III, 1999.

[11] Unger, S.H. and Tan, C.J. (1986) "Clocking schemes for high-speed digital systems", IEEE Transactions on Computers C-35(10), 880-895.

[12] Yeap, G.K. (1998) Practical Low Power VLSI design (Kluwer Academic Publishers, Dordrecht).

Dong Sam Ha received the B.S. degree in electrical engineering from Seoul National University, Korea, in 1974, and the M.S. and Ph.D. degrees in electrical engineering from the University of Iowa, Iowa City, in 1984 and 1986, respectively.Since Fall 1986, he has been a faculty member of the Bradley Department of Electrical Engineering, Virginia Polytechnic Institute and State University, Blacksburg. Currently, he is Professor with the department. Prior to his graduate study, he was a Research Engineer for the Agency for Defense Development in Korea from 1975 to 1979. While on leave from May to December of 1996, he was with the Semiconductor Research Center, Seoul National University, Seoul, Korea, where he investigated built-in self-test synthesis. Along with his students, he developed four computer-aided design tools for digital circuit testing. The source code for these four tools has been distributed to over 150 universities and research institutions worldwide, and the tools have been used for various research and teaching purposes at numerous universities.His research interests include low-power VLSI design for wireless communication, low-power analog and mixed-signal VLSI design, and low-power VLSI system design for wireless video. He can be reached atE-mail: ha@vt.edu.

Jos Budi Sulistyo was born in Jember, Indonesia. He obtained the B.S. degree in electrical engineering from Texas A\&M University 1993. Following his graduation, he joined the Indonesian National Atomic Energy Agency, at which he is currently a staff member. In August 1998, Jos joined Virginia Tech VLSI for Telecommunications (VTVT) group at the Bradley Department of Electrical and Computer Engineering, Virginia Polytechnic Institute and State University. Currently, he is enrolled in the Ph.D. program there. His current research interest is development of low power library cells including arithmetic units. He can be reached at E-mail: jsulisty@ee.vt.edu. 

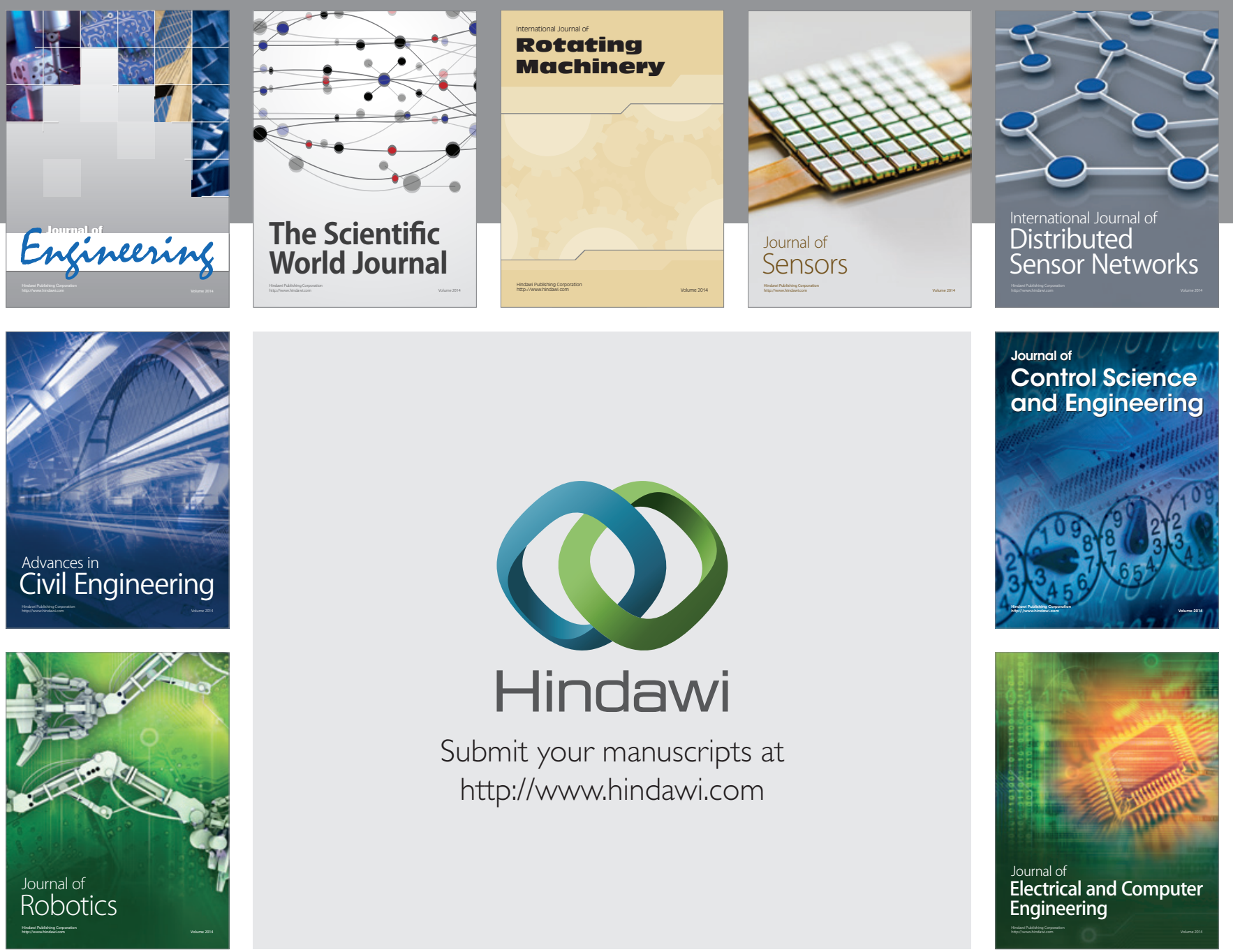

Submit your manuscripts at

http://www.hindawi.com
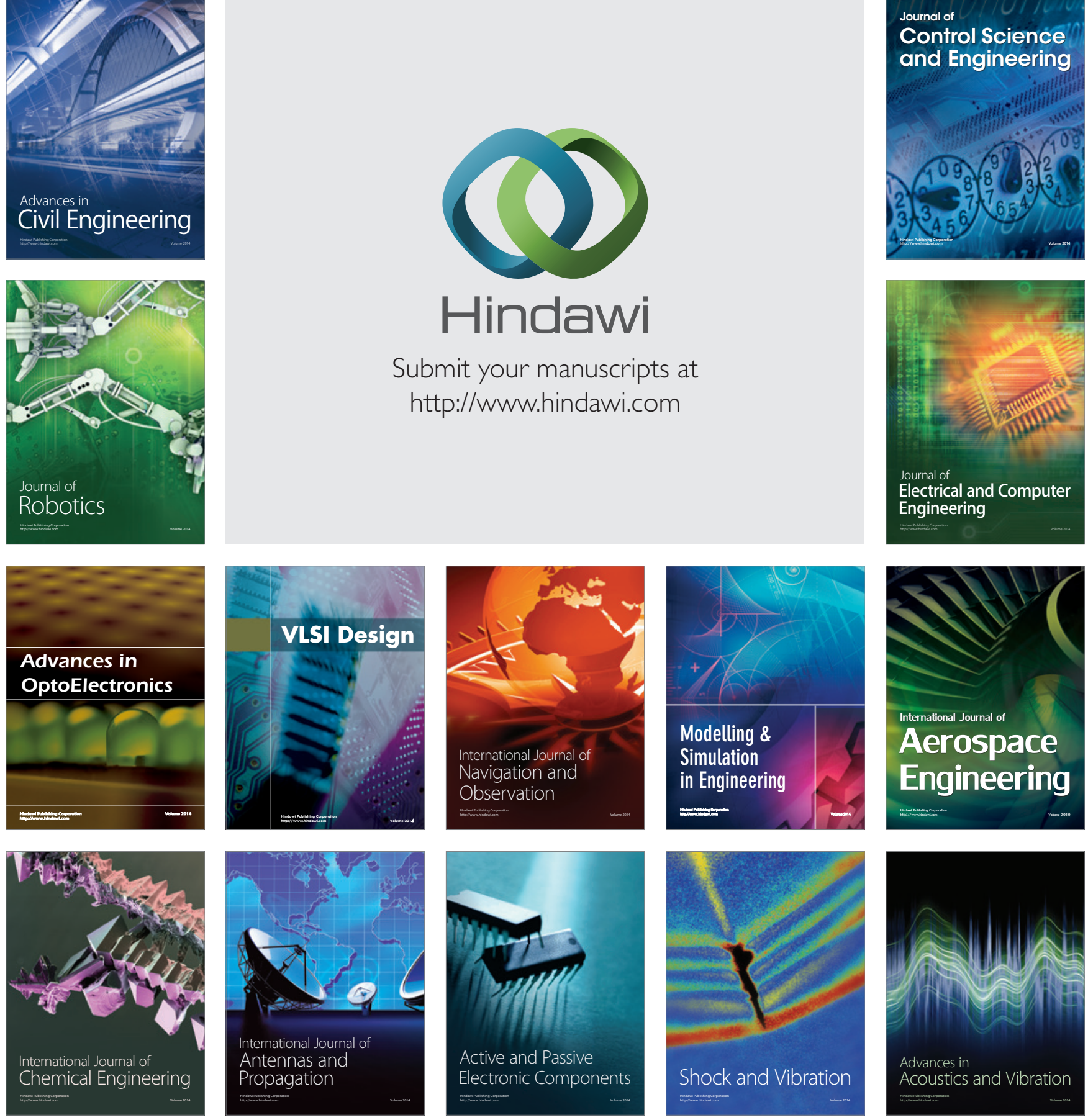